[0212-7199 (2007) 24: 9; pp 435-438] ANALES DE MEDICINA INTERNA Copyright (C) 2007 ARAN EDICIONES, S.L.

AN. MED. INTERNA (Madrid) Vol. 24, N. ${ }^{\circ} 9$, pp. 435-438, 2007

\title{
Síndrome de Young en la edad adulta: tratamiento domiciliario mediante un dispositivo mecánico de percusión intrapulmonar
}

\author{
E. RUESCAS ESCOLANO, E. CHINER VIVES, A. L. ANDREU RODRÍGUEZ, \\ A. CAMARASA ESCRIG, M. LLOMBART CANTÓ, J. SANCHO CHUST \\ Servicio de Neumología. Hospital Universitario San Juan de Alicante. Alicante
}

YOUNG'S SÍNDROME IN THE ADULT AGE: HOME TREATMENT WITH A MECHANICAL DEVICE OF INTRAPULMONARY PERCUSIVE VENTILATION

\begin{abstract}
RESUMEN
Se describe el caso de un varón afecto de bronquiectasias, infecciones rinosinusales de repetición y azoospermia obstructiva que fue diagnosticado de síndrome de Young en la edad adulta. Junto con las medidas convencionales, el paciente fue tratado en domicilio mediante un sistema mecánico de cuidados respiratorios consistente en ventilación por percusión intrapulmonar (Percussionaire ${ }^{\circledR}$ ) presentando una mejoría significativa clínica y funcional. Se señala la importancia de realizar una correcta historia de fertilidad en pacientes con clínica respiratoria compatible, con el fin de establecer el diagnóstico. Se revisan distintos métodos de asistencia mecánica respiratoria en pacientes con bronquiectasias, como complementarias en cuidado respiratorio domiciliario.
\end{abstract}

PALABRAS CLAVE: Síndrome de Young. Bronquiectasias. Esterilidad. Ventilación por percusión intrapulmonar. Percussionaire ${ }^{\circledR}$.

\begin{abstract}
We studied a man with bronchiectasis, chronic sinopulmonary infections and obstructive azoospermia daignosed of Young's syndrome in the adult age. The patient was treated at home by a mechanical system of respiratory care based in intrapulmonary percussive ventilation (Percussionaire ${ }^{\circledast}$ reaching a significant clinical and functional improvement. Our case enhanced the importance to make a proper history of fertility in patients with bronchiectasis in order to establish the diagnostic. We review different methods of respiratory mechanical asistance in patients with bronchiectasis.
\end{abstract}

KEY WORDS: Young's syndrome. Bronchiectasis. Sterility. Intrapulmonary percussive ventilation. Percussionaire ${ }^{\circledast}$.

Ruescas Escolano E, Chiner Vives E, Andreu Rodríguez AL, Camarasa Escrig A, Llombart Cantó M, Sancho Chust J. Síndrome de Young en la edad adulta: tratamiento domiciliario mediante un dispositivo mecánico de percusión intrapulmonar. An Med Interna (Madrid) 2007; 24: 435-438.

\section{INTRODUCCIÓN}

Son varias las enfermedades que asocian afectación nasosinusal, en forma de rinosinusitis, y bronquial en forma de bronquiectasias. Entre ellas, destaca la fibrosis quística, la discinesia ciliar primaria (DCP) y el déficit de alfa-1antitripsina (1).

En 1969, Young describió el síndrome que lleva su nombre $(2,3)$, para caracterizar a un subgrupo de pacientes que asocia enfermedad crónica pulmonar, azoospermia obstructiva con espermatogénesis normal y hallazgos característicos en el epidídimo, que constituye una rara causa de bronquiectasias en el adulto (4).

Presentamos el caso de un paciente afecto de síndrome de Young, que pese a reunir los hallazgos clínicos característicos del síndrome, fue diagnosticado en la edad adulta, y que como parte del tratamiento general, fue tratado con éxito mediante un nuevo sistema mecánico de cuidados respiratorios a domicilio (Percussionaire ${ }^{\circledR}$ ).

\section{CASO APORTADO}

Varón de 46 años de edad, marinero de profesión, casado y sin hijos, fumador de puros hasta hace 3 años, fue remitido a consulta de neumología por infecciones bronquiales de repetición.

Entre sus antecedentes destacaba la presencia de atelectasias timpánicas bilaterales durante la juventud, que precisaron drenajes transtimpánicos, con posterior caída progresiva de su audición, diagnosticada como hipoacusia neurosensorial bilateral. En los últimos cinco años, había presentado al menos 6-7 episodios anuales de

Trabajo aceptado: 16 de abril de 2007

Correspondencia: E. Chiner Vives. Sección de Neumología. Hospital Universitario San Juan de Alicante. Carretera Alicante-Valencia, s/n. 03550 San Juan de Alicante.E-mail: chiner_eus@gva.es 
infecciones de vías bajas, y tres episodios de neumonía. Asimismo, refería congestión nasal prácticamente permanente, disminución del olfato y episodios remotos compatibles con sinusitis. En los dos últimos años presentaba disnea a esfuerzos moderados y broncorrea habitual.

Tres días antes de su evaluación, el paciente había presentado fiebre de $38{ }^{\circ} \mathrm{C}$ con aumento de la expectoración basal, de coloración blanquecina, acompañada de dolor en ambos hemitórax de características pleuríticas, así como disnea a moderados esfuerzos. A la exploración física, destacaba la presencia de crepitantes bibasales.

La radiografía de tórax mostró un infiltrado intersticio-alveolar bilateral e imágenes de bronquiectasias de predominio en lóbulo inferior izquierdo (Fig. 1 a y b). La TACAR evidenció bronquiectasias bibasales de predominio izquierdo y múltiples nódulos acinares de predominio parahiliar con área en vidrio deslustrado en base derecha y zona de mayor condensación en base izquierda (Fig. 2). En los análisis sanguíneos no se observaron alteraciones, con un nivel de proteínas totales, alfa-1-antitripsina, IgG, IgM e IgA normales. La exploración funcional respiratoria mostró TLC $4.850 \mathrm{ml}$ (69\%), RV $1.830 \mathrm{ml}$ (88\%), FVC de $3.010 \mathrm{ml}$ (58\%), FEV1 de 1.920 $\mathrm{ml}(48 \%)$ y una relación FEV1/FVC de 64 (84\%), compatible con un trastorno ventilatorio de tipo mixto de predominio obstructivo en grado moderado con test broncodilatador negativo. La fibrobronco-
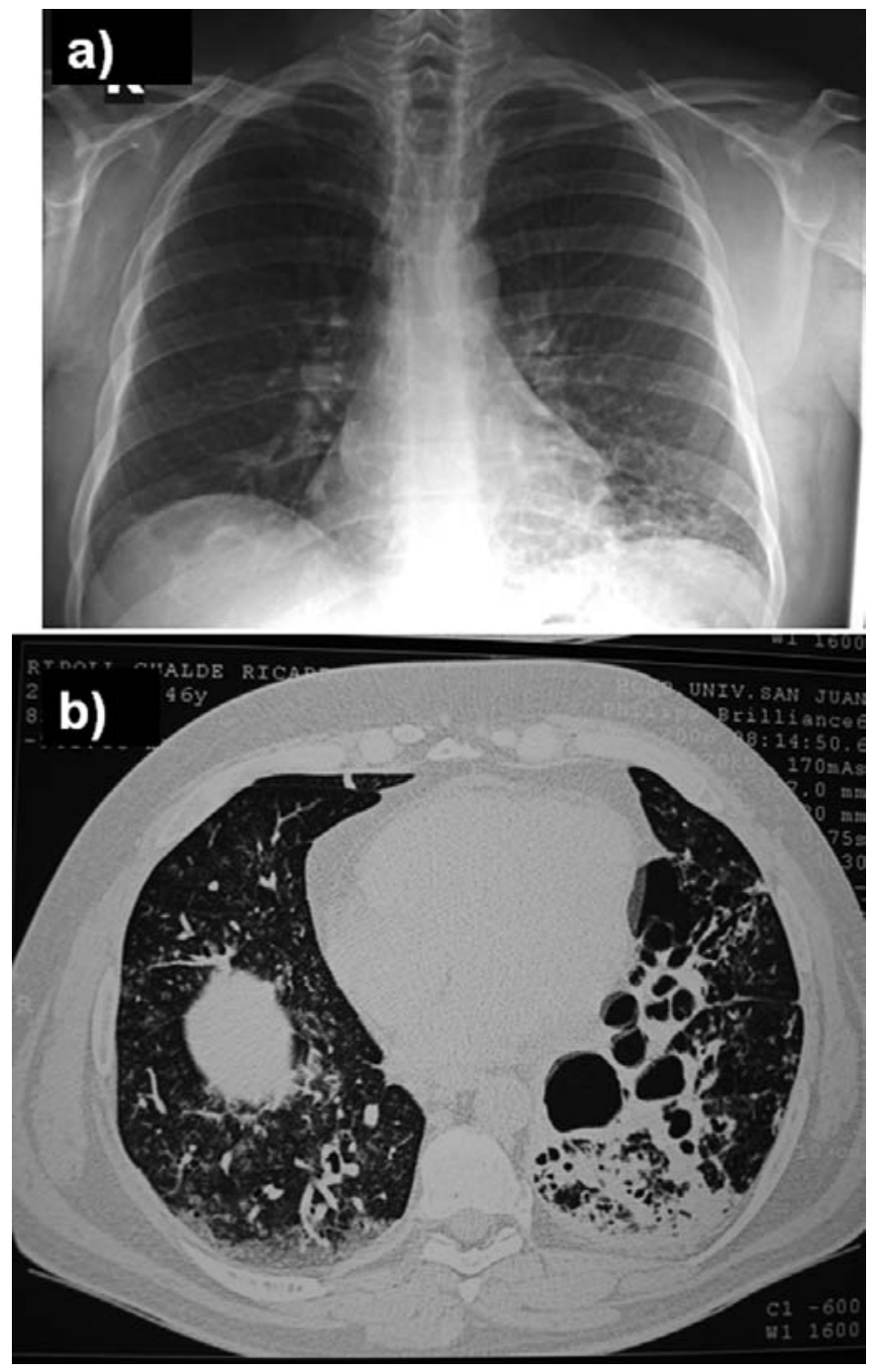

Fig. 1. a) Radiografía postero-anterior de tórax que muestra imágenes compatibles con bronquiectasias en ambos lóbulos inferiores. b) Imagen de TAC mostrando bronquiectasias quísticas y zonas de condensación en ambos lóbulos inferiores.
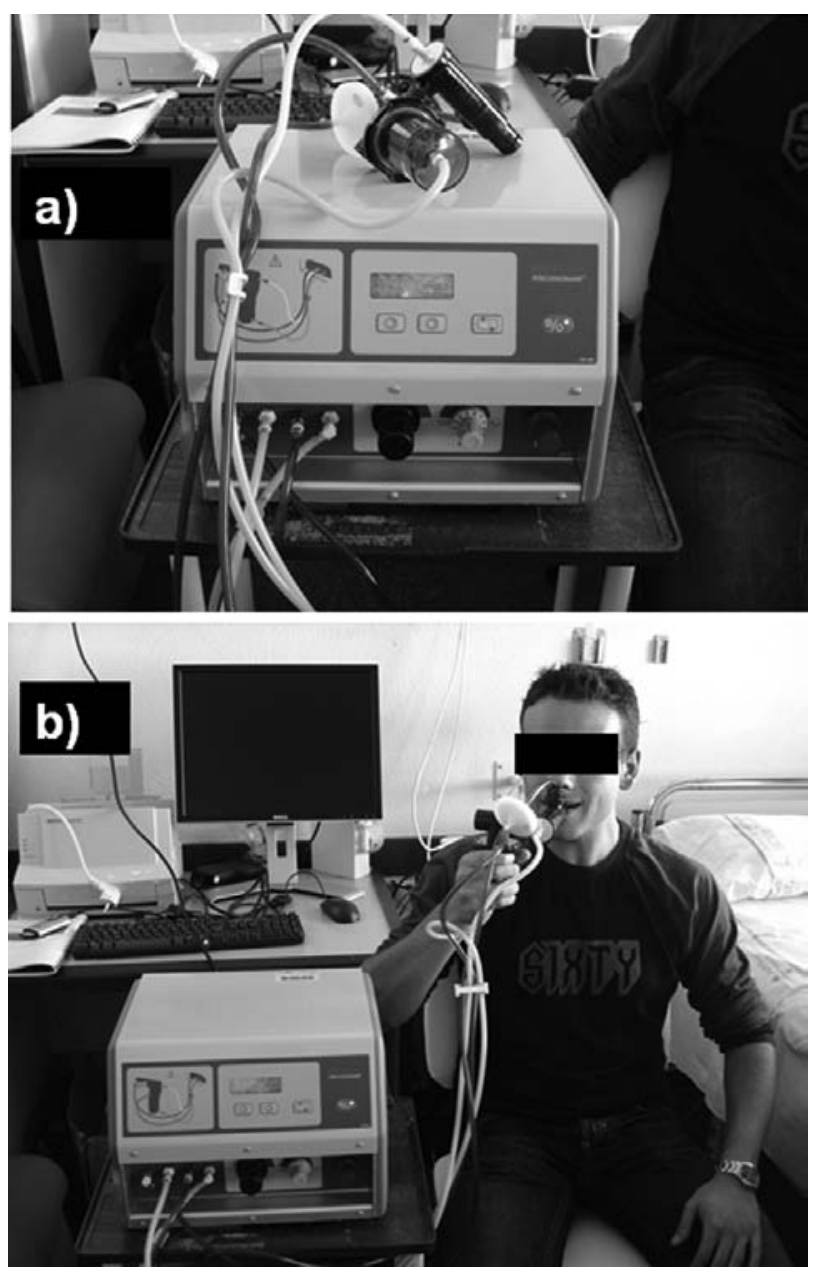

Fig. 2. a) Dispositivo mecánico de percusión intrapulmonar (Percussionaire ${ }^{\circledast}$. b) Utilización del dispositivo en el paciente.

scopia mostró signos indirectos de bronquiectasias y lesiones de broncopatía crónica, abundantes secreciones purulentas y trastorno malácico en bronquio principal izquierdo. El cultivo y Zhiel de esputo, broncoaspirado (BAS) y lavado broncoalveolar (BAL) fueron negativos. En el catéter telescopado se aisló Pseudomonas aeruginosa sensible a ceftacidima, gentamicina, amikacina, ciprofloxacino, cotrimoxazol y fosfomicina. La citología del BAS y BAL fue negativa para células malignas. Los hemocultivos fueron negativos.

Se realizó un estudio de transporte mucociliar nasal isotópico que mostró ausencia de respuesta tras 30 minutos de observación. El test del sudor fue normal.

Al paciente se le propuso la realización de un espermiograma para valorar de manera indirecta la motilidad ciliar y en ese momento refirió que había sido valorado quince años antes como parte de un estudio de esterilidad conyugal, siendo diagnosticado de azoospermia obstructiva, con ausencia de cuerpo y cola del epidídimo, que se intentó corregir mediante tubulovasostomía bilateral sin éxito.

Se inició tratamiento con oxígeno, broncodilatadores, esteroides inhalados y antibioterapia empírica mediante ceftacidima y posteriormente tobramicina aerosolizada en domicilio a dosis de $300 \mathrm{mg}$ cada 12 horas en pauta secuencial. A su vez se realizó fisioterapia respiratoria y se instruyó al paciente en técnicas de drenaje postural. Estas medidas fueron complementadas mediante la aplicación de un sistema de ventilación percutoria intrapulmonar (Percussionaire ${ }^{\circledR}$ ) en 
sesiones diarias de mañana, tarde y noche, de media hora de duración, con una presión de operación de $20 \mathrm{~cm}$ de $\mathrm{H} 2 \mathrm{O}$ a una frecuencia de 250 ciclos por minuto, siendo pautado a domicilio. Dos meses después se observó una disminución significativa del volumen de secreciones diarias y la exploración funcional respiratoria mostró TLC $5.770 \mathrm{ml}$ (81\%), RV $1.730 \mathrm{ml}$ (82\%), FVC $3.450 \mathrm{ml}(65 \%)$, FEV1 $2.390 \mathrm{ml}(59 \%)$ y una relación FEV1/FVC de 69 (91\%), lo que supuso un incremento del 15\% en FVC y del $24 \%$ en FEV1.

\section{DISCUSIÓN}

Aunque Young describió en 1969 los rasgos clínicos característicos del síndrome (2,3), no es hasta 1984 en que Handelsman y colaboradores, estudian una población de 29 varones que combinan infecciones crónicas sinopulmonares y azoospermia obstructiva. La edad media en el momento del diagnóstico era de 31 años y presentaban solamente una leve alteración de la función respiratoria con espermatogénesis normal, siendo debida la azoospermia a la obstrucción del epidídimo por el espesamiento de las secreciones, estimando su prevalencia entre hombres no fértiles comparable a la del síndrome de Klinefelter, pero superior a la de la fibrosis quística o el síndrome de cilio inmóvil (4).

La azoospermia causante de la infertilidad se debe a la obstrucción intraluminal del epidídimo, probablemente progresiva, debido a la viscosidad de los fluidos, ya que la espermiogénesis es totalmente normal. La obstrucción puede no llegar a ser completa hasta años después de la pubertad. En muchos casos se observa la dilatación de la cabeza del epidídimo llena de espermatozoides y presencia de material amorfo sin espermatozoides a nivel del cuerpo $(4,5)$.

Una característica importante es que la actividad y la estructura ciliar es normal a diferencia de lo que ocurre en la DCP. Por otro lado, el moco tiene incrementada su viscosidad, de modo que la clínica resultante es superponible a las patologías que entran dentro del diagnóstico diferencial (1). La biopsia nasal bajo microscopia electrónica no muestra cambios en la estructura de los cilios (6).

En el síndrome de Young no se observan las alteraciones típicas de los síndromes de DCP (alteración de los brazos de dineína y/o disposición anómala de los microtúbulos que ocasionan la disfunción parcial o total de las células ciliadas y flageladas), ni las características electrolíticas y genéticas típicas de la fibrosis quística (7) (iontoforesis con pilocarpina con una concentración de cloro superior a $60 \mathrm{mmol} / 1$ en dos muestras separadas, o mutación del factor regulador de la fibrosis quística, siendo típica la DF508), ni tampoco las mutaciones genéticas de la ausencia congénita del conducto deferente, que justifica un 6\% de los casos de azoospermia obstructiva. De esta forma, se establece que el síndrome de Young es una entidad clínica diferente a las otras enfermedades (7).

Como en nuestro caso, el síndrome de Young se debe descartar en varones con síntomas sinopulmonares recidivantes crónicos e infertilidad. El diagnóstico se basa en la clínica, así como en la exclusión de fibrosis quística y de síndromes de inmovilidad ciliar (4). En este sentido, la anamnesis dirigida hacia la historia de fertilidad es importante, pues puede prácticamente establecer el diagnóstico, y en ocasiones ésta puede pasar desapercibida.

Recientemente, en un estudio con 150 adultos con bronquiectasias se ha señalado que realizar una investigación intensa en estos pacientes para intentar llegar a un diagnóstico etiológico, permite identificar una o más causas en el $47 \%$ de los casos y que en el 15\%, la causa identificada tiene implicaciones en el tratamiento y en el pronóstico (8).

Se han publicado algunos casos como variantes del síndrome de Young en los que, aparte de la triada clínica típica, aparece situs inversus, una característica más típica del síndrome de Kartagener. En uno de los casos publicados se observa, mediante microscopio electrónico, alteraciones en el $65 \%$ de los microtúbulos centrales de los cilios nasales, atrofia del cuerpo del epidídimo y motilidad y estructura del esperma normales (9). En otro estudio más reciente, se describen dos casos con defectos parciales en los brazos de la dineína y se apunta la hipótesis del origen genético similar para ambos síndromes (10). Es posible que estos casos sean entidades distintas.

El abordaje terapéutico clásico de las bronquiectasias, tanto en niños como en adultos consiste, por una parte, en la adopción de medidas generales, como son el tratamiento antibiótico enérgico de las infecciones de repetición, la vacunación contra la gripe, el uso de broncodilatadores y recientemente se ha incorporado a la terapia los esteroides inhalados. Por otra parte, la fisioterapia respiratoria para facilitar la eliminación de secreciones se considera fundamental, si bien la terapia clásica consistente en medidas de drenaje postural, percusión, tos provocada y dirigida, vibración y técnicas de respiración forzada (TEF) o "huffing", se ha puesto en entredicho (11).

En los últimos años, se han desarrollado ayudas mecánicas para facilitar el cumplimiento de la fisioterapia por parte del paciente, ya que muchos enfermos suelen abandonar las técnicas convencionales (12). Entre las técnicas mecánicas de ayuda destaca la presión espiratoria positiva oscilante a alta frecuencia, de la que existen dos modalidades denominadas Flutter $^{\circledR}$ y Acapella ${ }^{\circledR}$. Flutter ${ }^{\circledR}$, es un pequeño dispositivo portátil que produce de forma controlada ondas de presión positiva oscilante mediante el desplazamiento repetido de una bola de acero por la fuerza de la gravedad, en el interior de un cono de plástico. Al comparar el Flutter $^{\circledR}$ con los ciclos activos de técnicas respiratorias (CATR), que incluye un conjunto de técnicas aplicadas de forma secuencial para facilitar la movilidad de secreciones (respiración controlada, expansión torácica y maniobras de espiración forzada), en pacientes con bronquiectasias no debidas a fibrosis quística, parece que no existe diferencia en los resultados clínicos obtenidos entre ambas. De esta forma, el Flutter ${ }^{\circledR}$ sería tan efectivo en la eliminación de secreciones como los CATR, siendo bien tolerado, sin efectos adversos, no requiriendo mayor tiempo para ser efectivo y con un alto nivel de aceptabilidad (13).

Acapella ${ }^{\circledR}$ utiliza el contrapeso de una palanca y la fuerza de un imán para crear las oscilaciones de forma independiente de la gravedad. Al comparar esta técnica con CATR no hay diferencias clínicas entre ambos sistemas de tratamiento y la mayoría de pacientes mostraron preferencia por Acapella ${ }^{\circledR}$, por lo que ésta constituye una alternativa eficiente para el tratamiento de las bronquiectasias (14). Al comparar Acapella $^{\circledR}$ con Flutter ${ }^{\circledR}$, mostraron un funcionamiento similar y sus diferencias no fueron clínicamente significativas; Acapella ${ }^{\circledR}$ crea oscilaciones más estables en amplitud y frecuencia y genera oscilaciones amplias con flujos espiratorios muy bajos (5L/min), por lo que resulta más útil en casos de obstrucción pulmonar grave (15). 
En nuestro paciente utilizamos un dispositivo de Ventilación por Percusión Intrapulmonar (FM Bird, Percussionaire $^{\circledR}$ Corporation, Sandpoint, Idaho). Se trata de una técnica de tratamiento y de ventilación en patologías respiratorias obstructivas y/o restrictivas mayores, consistente en la administración de un flujo pulsátil de gas dentro de los pulmones del paciente, a una frecuencia alta, a través de un circuito respiratorio abierto. Las percusiones suministran pequeños volúmenes de gas, inferiores al volumen corriente, que varían entre 1 y $300 \mathrm{cc}$, que son suministrados de manera continua y pulsátil a la vía aérea del paciente, superponiéndose o no, a la ventilación espontánea. El circuito respiratorio abierto denominado Phasitron ${ }^{\circledR}$ es un conversor de flujo/presión que convierte volúmenes de bajo flujo y alta presión en volúmenes de alto flujo y baja presión y está asociado a un nebulizador neumático. Este sistema se adapta a las características clínicas del paciente, de tal forma que se consigue la mayor adherencia posible al mecanismo. Este sistema se ha usado tanto en niños como en adultos con atelectasias refractarias a la terapia convencional (16) y en pacientes con fibrosis quística (17). El sistema permite acoplar un nebulizador como complemento a la fisioterapia suministrada por el aparato (18). En nuestro caso fue bien tolerado y se observó con el uso continuado del mismo, junto con el tratamiento médico, una disminución significativa de las secreciones y una mejoría en la función pulmonar.

En conclusión, queremos resaltar, en primer lugar, la importancia de investigar la historia de fertilidad en todo varón adulto que presenta bronquiectasias, dada la posibilidad de encontrarnos ante un síndrome de Young o un caso de DCP, de cara a plantear el tratamiento médico y quirúrgico y la posibilidad de consejo genético. En segundo lugar, destacar el avance en las técnicas de ayuda mecánica para drenaje de secreciones bronquiales, tales como el Percussionaire ${ }^{\circledast}$ que parecen tener buenos resultados y son bien tolerados y aceptados por los pacientes que los utilizan.

\section{Bibliografía}

1. Guilemany JM, Mullol J, Picado C. Relation between rhinosinusitis and bronchiectasis. Arch Bronconeumol 2006; 42: 135-40.

2. Young D. Cystic fibrosis and fertility. Br Med J 1969; 3: 594.

3. Young D. Surgical treatment of male infertility. J Reprod Fertil 1970; 23: 541-542.

4. Handelsman DJ, Conway AJ, Boylan LM, Turtle JR. Young's syndrome: Obstructive azoospermia and chronic sinopulmonary infections. N Engl J Med 1984; 310: 3-9.

5. Lau KY, Lieberman J. Young's syndrome. An association between male sterility and bronchiectasis. West J Med 1986; 144: 744-6.

6. Domingo C, Mirapeix RM, Encabo B, Roig J, López D, Ruiz J. Hallazgos clínicos y ultraestructura de la discinesia ciliar primaria y el síndrome de Young. Rev Clin Esp 1997; 197: 100-3.

7. Le Lannou D, Jezequel P, Blayau M, Dorval I, Lemoine P, Dabadie, et al. Obstructive azoospermia with agenesis of the vas deferens or with bronquiectasia (Young's syndrome): a genetic approach. Hum Reprod 1995; 10: 338-41.

8. Pasteur MC, Helliwell SM, Houghton SJ, Webb SC, Foweraker JE, Coluden RA, et al. An investigation into causative factors in patiens with bronchiectasis. Am J Respir Crit Care Med 2002; 162: 1277-1284.

9. Shiraishi K, Ono N, Mohri J, Kamiryo Y, Takihara H. Young's syndrome associated with inversus totalis. Arch Androl 2004; 50: 169-72.

10. Ichioka K, Kohei N, Okubo K, Nishiyama H, Terai A. Obstructive azoospermia associated with chronic sinopulmonary infection and situs inversus totalis. Urology 2006; 68: 204.e5-7.

11. American Association of Cardiovascular and Pulmonary Rehabilita-

tion. Guidelines for pulmonary rehabilitation programs. $3^{\mathrm{a}}$ ed. United States: Human Kinetics; 2004.

12. Morales P, Morant P, Solé A, Lozano C. Rehabilitación Respiratoria en fibrosis quística y bronquiectasias. En: Güell R, De Lucas P, editores. Rehabilitación Respiratoria. Madrid: EGRAF, S.A.; 1999. p. 253-267.

13. Thompson CS, Harrison S, Ashley J, Day K, Smith DL. Randomised crossover study of the Flutter device and the active cycle of breathing technique in non-cystic fibrosis bronchiectasis. Thorax 2002; 57: 446448 .

14. Patterson JE, Bradley JM, Hewitt O, Bradbury I, Elborn JS. Airway clearance in bronchiectasis: a randomized crossover trial of active cycle of breathing techniques versus Acapella. Respiration 2005; 72: 239342.

15. Volsko TA, DiFiore J, Chatburn RL. Performance comparison of two oscillating positive expiratory pressure devices: Acapella versus Flutter. Respir Care 2003; 48: 124-30

16. Birnkrant DJ, Pope JF, Lewarski J, Stegmaier J, Besunder JB. Persistent pulmonary consolidation treated with intrapulmonary percussive ventilation: a preliminary report. Pediatr Pulmonol 1996; 21: 246-9.

17. Fink JB, Mahlmeister MJ. High-frequency oscillation of the airway and chest wall. Respir Care 2002; 47: 729-807.

18. Reychler G, Keyeux A, Cremers C, Veriter C, Rodenstein DO, Liistro G. Comparison of lung deposition in two types of nebulization: intrapulmonary percussive ventilation vs jet nebulisation. Chest 2004; 125 : $502-8$. 